\section{Cranioplasty Sinking Should Affect Normal Brain Function Mimicking Other Neurologic Illness}

We present the case of a 62-year-old man, with a history of severe head injury, right parietal fracture, frontal concussion, right subdural hematoma, and posttraumatic hydrocephalus. Two months later he showed progressive neurologic deterioration with bradypsychia, loss of mobility of the left side of the body, a decreased level of consciousness, as well as a speech disorder coinciding with a sinking cranioplasty (Fig 1A). This deterioration progressed despite having a similar ventricular size and an apparent normofunctioning valve. FDG-PET was performed, showing a marked right hemispheric hypometabolism and hypometabolism of the head of the right caudate and thalamus (Figs $1 B$ and $1 C$ ), compared with the primary motor, auditory, and cingulate-posterior cortex. There was also a marked decrease in left cerebellar metabolism, probably related to deafferentation. This FDG-PET finding, despite a history of head injury, raises a differential diagnosis with corticobasal degeneration or frontotemporal dementia, both diseases related to deposition of hyperphosphorylated $\tau$ protein.

The inhibition of function in a portion of the brain at a distance from the original site of injury is known as "diaschisis." In the presented case, these neurologic deficits may be a consequence of reduced cerebral blood flow ${ }^{1}$ and a disturbed metabolism due to direct cortical compression of the sinking cranioplasty and the secondary diaschisis at different levels (corticocerebellar, thalamic, striatal, and cortical).
Neurosurgical assessment of the ventriculoperitoneal valve (malfunctioning) and replacement of the cranioplasty (Fig 1D) were performed. The patient improved significantly, and the PET pattern changed (Figs $1 E$ and $1 F$ ), showing only frontal hypometabolism, both dorsolateral and dorsomedial, probably related to the known hydrocephalus. The observed changes suggest that there is a hemodynamic problem ${ }^{2}$ giving rise to an impaired cerebral metabolism when a sinking cranioplasty affects the cerebral cortex and that it can improve after cranioplasty replacement, ${ }^{3}$ which also reflects the plasticity of the human brain.

\section{References}

1. Kemmling A, Duning T, Lemcke L, et al. Case report of MR perfusion imaging in sinking skin flap syndrome: growing evidence for hemodynamic impairment. BMC Neurology 2010;10:80

2. Sakamoto S, Eguchi K, Kiura Y, et al. CT perfusion imaging in the syndrome of the sinking skin flap before and after cranioplasty. Clin Neurol Neurosurg 2006;108L583-85

3. Isago T, Nozaki M, Kikuchi Y, et al. Sinking skin flap syndrome: a case of improved cerebral blood flow after cranioplasty. Ann Plast Surg 2004;53: $288-92$

M. del Mar Carmona Abellán

M. Murie Fernández

P. Esteve Belloch

Department of Neurology

Clinica Universidad de Navarra

Pamplona, Spain

http://dx.doi.org/10.3174/ajnr.A3094

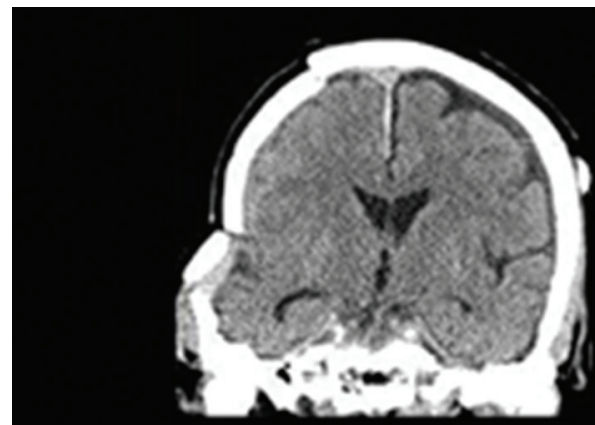

A

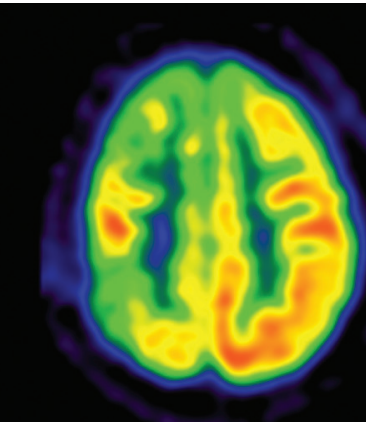

B

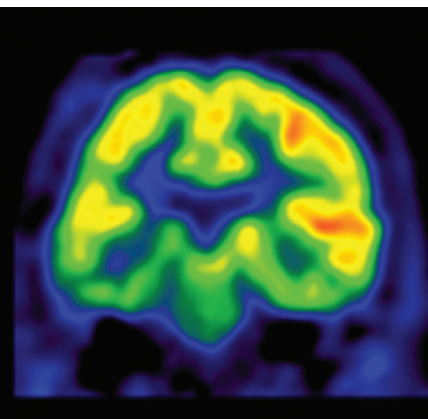

C

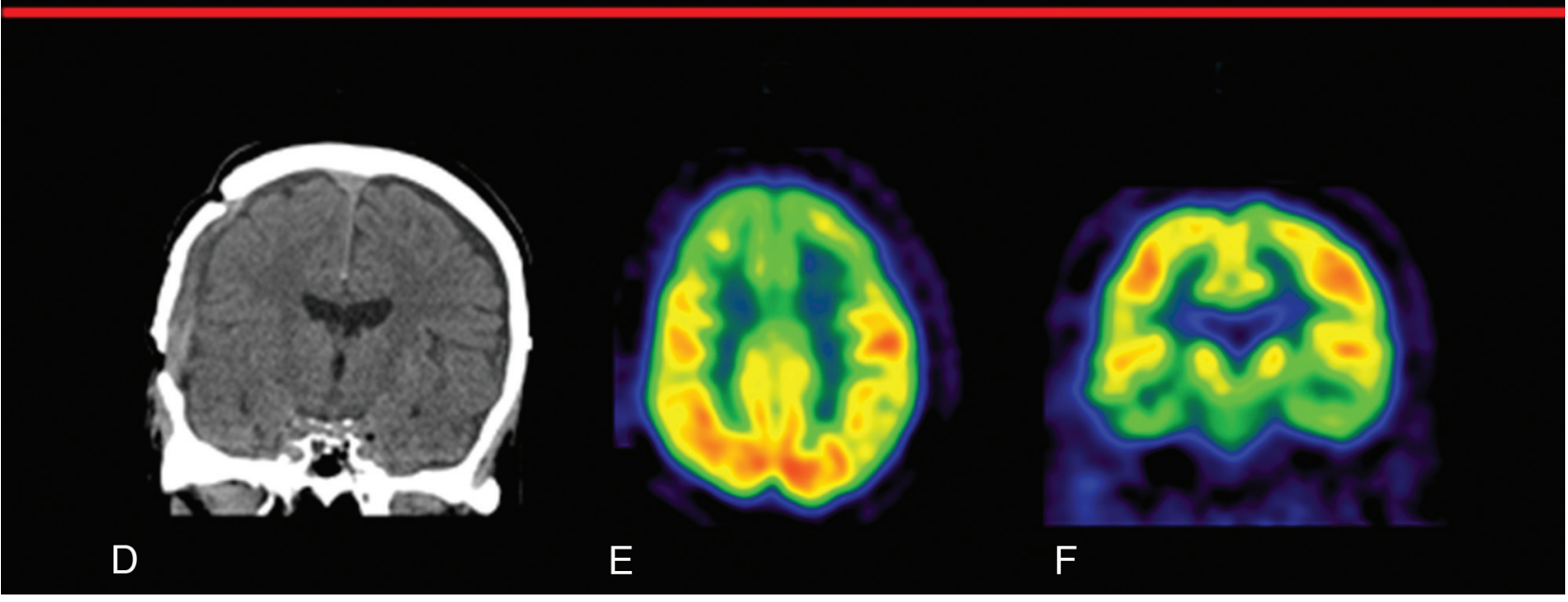

Fig 1. $A$, Sinking cranioplasty. $B$ and $C$, Right hemispheric hypometabolism, more evident in the frontal, parietal and temporal cortex, and affecting anterior cingulate. There is a marked hypometabolism of the head of the caudate and right thalamus and in left cerebellar metabolism. $D$, Replacement of the cranioplasty. $E$ and $F$, Frontal hypometabolism both dorsolateral and dorsomedial. 